\title{
Description of Extreme Gibbs Measures for the Ising Model with Three Interactions
}

\author{
H. Akin ${ }^{a}$, N. Ganikhodjaev ${ }^{b}$, S. TemiR ${ }^{c}$ And S. UGuZ \\ ${ }^{a}$ Zirve University, Faculty of Education, Department of Mathematics, Gaziantep, 27260, Turkey \\ ${ }^{b}$ Department of Computational and Theoretical Sciences, Faculty of Science, IIUM, 25200 Kuantan, Malaysia \\ ${ }^{c}$ Harran University, Arts and Science Faculty, Department of Mathematics, Sanliurfa, 63120, Turkey \\ In this paper, we consider an Ising model with three competing interactions (nearest neighbor, next-nearest \\ neighbor, and ternary prolonged neighbor) on the Cayley tree of order two, investigated by Ganikhodjaev et al. \\ We study translation-invariant Gibbs measures of the Ising model with these competing interactions. Also, we \\ investigate the set of the extreme Gibbs measures called Markov random fields with memory 2 of the model.
}

DOI: 10.12693/APhysPolA.123.484

PACS: 05.50.+q, 64.60.-i, 64.60.De, 75.10.Hk

\section{Introduction and definitions}

One of the central objects of equilibrium statistical mechanics is the Gibbs measure [1], a branch of probability theory that takes its origin from Boltzmann [2]. Also, one of the main problems of the statistical physics is to describe all the Gibbs measures corresponding to the given Hamiltonian. It is well known that such measures form a nonempty convex compact subset in the set of all probabilistic measures. The purpose of this paper is to investigate the Gibbs measures of the Ising model [3] with ternary prolonged and nearest neighbor interactions on the Cayley tree of order two and to describe its extreme elements (pure phases). In [4], we have studied phase diagram and extreme Gibbs measures of the Ising model on a Cayley tree in the presence of competing binary and ternary interactions. In [5], we have obtained the extreme Gibbs measures of the Vannimenus model [6]. We studied the Gibbs states (phases) that correspond in probability theory to what are called Markov chains with memory length 2 by using the method in [7]. In this paper, we combine the results obtained in [4] and [5]. Our model with competing nearest-neighbors, next-nearest neighbors, and prolonged next-nearest-neighbors ternary interactions is defined by the following Hamiltonian:

$$
\begin{aligned}
& H(\sigma)=-J_{1} \sum_{\langle x, y\rangle} \sigma(x) \sigma(y)-J_{p} \sum_{\rangle \widetilde{x, z}\langle} \sigma(x) \sigma(z) \\
& -J_{t} \sum_{\widehat{x, y, z}\langle} \sigma(x) \sigma(y) \sigma(z)
\end{aligned}
$$

where the sum in the first term ranges nearest-neighbors $(\mathrm{NN})$, the sum in the second term ranges all next-nearest neighbors (NNN) and the sum in the third term ranges all prolonged ternary next-nearest-neighbors (PTNNN). Here $J_{1}, J_{p}, J_{t} \in R$ are coupling constants (see [3] for details). As usual, one can introduce the notions of the Gibbs distribution of this model, limiting Gibbs distribution, pure phase (extreme Gibbs distribution), etc. (see [7-9]). See [3] for a model (1) producing basic equations.

Definition 2.1. Let $\left\langle x^{0}, x^{1}\right\rangle=l_{0} \in L$ be an edge of the Cayley tree $\Gamma^{k}$. The infinite subtree $\Gamma_{l_{0}}^{k}=\left(V^{l_{0}}, L^{l_{0}}\right)$ is called a single-trunk Cayley tree, if from vertex $x^{0}$ single edge $l_{0}$ emanates and from any other vertex $x \in$ $V^{l_{0}}, x \neq x^{0}$ exactly $k+1$ edges emanate. If an arbitrary edge $\left\langle x^{0}, x^{1}\right\rangle=l_{0} \in L$ is deleted from the Cayley tree $\Gamma^{k}$, it splits into four components four single-trunk Cayley trees $\Gamma_{l_{i}}^{k}, i=1,2,3,4$, where $i\left(l_{1}\right)=\left\langle x^{0}, x^{2}\right\rangle, i\left(l_{2}\right)=$ $\left\langle x^{0}, x^{3}\right\rangle, i\left(l_{3}\right)=\left\langle x^{1}, x^{4}\right\rangle, i\left(l_{4}\right)=\left\langle x^{1}, x^{5}\right\rangle$.

Theorem 2.1. In order for $\mu$ to be an extreme Gibbs distribution on $\Gamma^{k}$, it is necessary and sufficient that there exist extreme Gibbs distributions $\mu_{i}, i=1,2,3,4$ (which are determined uniquely by $\mu$ ) on $\Gamma_{l_{i}}^{k}, i=1,2,3,4$, respectively, such that

$$
\begin{aligned}
\mu & =\prod_{i=1}^{4} \mu_{i} Z^{-1}\left[\exp \left(\beta J_{1} \sigma\left(x^{0}\right) \sigma\left(x^{1}\right)\right)\right. \\
& +\exp \left(\beta J_{t} \sigma\left(x^{1}\right) \sigma\left(x^{0}\right) \sigma\left(x^{4}\right)\right) \\
& +\exp \left(\beta J_{t} \sigma\left(x^{1}\right) \sigma\left(x^{0}\right) \sigma\left(x^{5}\right)\right) \\
& +\exp \left(\beta J_{t} \sigma\left(x^{0}\right) \sigma\left(x^{1}\right) \sigma\left(x^{2}\right)\right) \\
& +\exp \left(\beta J_{t} \sigma\left(x^{0}\right) \sigma\left(x^{1}\right) \sigma\left(x^{3}\right)\right) \\
& +\exp \left(\beta J_{p} \sigma\left(x^{1}\right) \sigma\left(x^{4}\right)\right)+\exp \left(\beta J_{p} \sigma\left(x^{1}\right) \sigma\left(x^{5}\right)\right) \\
& \left.+\exp \left(\beta J_{p} \sigma\left(x^{0}\right) \sigma\left(x^{2}\right)\right)+\exp \left(\beta J_{p} \sigma\left(x^{0}\right) \sigma\left(x^{3}\right)\right)\right]
\end{aligned}
$$

where $Z>0$ is the normalizing constant and $\beta=1 / T$ is the inverse temperature. Theorem 2.1 reduces the description of extreme Gibbs distributions on $\Gamma^{k}$ to the single-trunk Cayley tree $\Gamma_{l_{0}}^{k}$. Let $V^{l_{0}}$ be the set of vertices of the $\Gamma_{l_{0}}^{k}$ and $L^{l_{0}}$ its set of edges. On the tree $\Gamma_{l_{0}}^{k}$ one can single out the boundary vertex $x^{0} \in V^{l_{0}}$ from which single edge $l_{0}=\left\langle x^{0}, x^{1}\right\rangle \in L^{l_{0}}$ emanates. As above we set $W_{n}^{l_{0}}=\left\{x \in V^{l_{0}} \mid d\left(x, x^{0}\right)=n\right\}, V_{n}^{l_{0}}=\{x \in$ $\left.V^{l_{0}} \mid d\left(x, x^{0}\right) \leq n\right\}$, and $L_{n}^{l_{0}}$ be the set of edges in $V_{n}^{l_{0}}$. It is evident that $\left|W_{0}^{l_{0}}\right|=\left|W_{1}^{l_{0}}\right|=1$ and $\left|W_{n}^{l_{0}}\right|=2^{n-1}$ for $n \geq 2$. We write $l_{1} \prec l_{2}$ if $i\left(l_{1}\right)=\langle x, y\rangle, i\left(l_{2}\right)=\langle z, t\rangle$ and $x \prec y \prec z \prec t$. We call the edge $l_{2}$ a direct successor of $l_{1}$ if $i\left(l_{1}\right)=\langle x, y\rangle, i\left(l_{2}\right)=\langle y, z\rangle$ and $x \prec y \prec z$. For given edge $l_{x}=\langle x, y\rangle \in L^{l_{0}}$ with $x \prec y$ the set 
of vertices $V_{l}^{l_{0}}=\left\{x, y, z \in V^{l_{0}} \mid z \succ y\right\}$ with the edges connecting them form the single-trunk Cayley tree $\Gamma_{l_{x}}^{k}$ "growing" from the edge $l_{x}=\langle x, y\rangle \in L^{l_{0}}$.

Theorem 2.2. Let $n \geq 2$. In order for $\mu$ to be an extreme Gibbs distribution on $\Gamma_{l_{0}}^{k}$, it is necessary and sufficient that there exist extreme Gibbs distribution $\mu_{l_{x}}$ on $\Gamma_{l_{x}}^{k}, x \in W_{n}^{l_{0}}$ (which are uniquely determined by $\mu$ ) such that

$$
\mu=Z_{n}^{-1} \exp \left(-\beta H_{n}(\sigma)\right) \prod_{x \in W_{n}} \mu_{l_{x}},
$$

where

$$
\begin{aligned}
& H_{n}(\sigma)=-J_{t} \sum_{\substack{y, y, y \backslash \\
x, y, z \in V_{n}}} \sigma(x) \sigma(y) \sigma(z) \\
& -J_{p} \sum_{\substack{\lambda \widetilde{x, z}\left\langle \\
x, z \in V_{n}\right.}} \sigma(x) \sigma(z)-J_{1} \sum_{\substack{\langle x, y\rangle \\
x, y \in V_{n}}} \sigma(x) \sigma(z) .
\end{aligned}
$$

Proof: This also follows from Theorem III.1 [7].

Denote as above by $E=E\left(\Gamma_{l_{0}}^{k}\right)$ the set of extreme Gibbs distributions on $\Gamma_{l_{0}}^{k}$ and by $F=F\left(\Gamma_{l_{0}}^{k}\right)$ the set of the Gibbs distributions on $\Gamma_{l_{0}}^{k}$ such that there exist the Gibbs distributions $\mu_{l_{x}}$ on $\Gamma_{l_{x}}^{k}, x \in W_{n}^{l_{0}}$ such that for each $n \geq 0$ the factorization (2) is true. From Theorem 3.2 it follows that $E \subset F$. The class $F$ is the class of the Markov random fields on the Cayley tree (see [8-10]).

Theorem 2.3. Let $\mu \in F$ and $\left\{\mu_{l_{x}}, x \in V^{l_{0}}\right\}$ be the corresponding Gibbs distributions on $\Gamma_{l_{x}}^{k}, x \in V^{l_{0}}$. Then, for any $x \in V^{l_{0}}, \mu_{l_{x}} \in F\left(\Gamma_{l_{x}}^{k}\right)$ and $\left\{\mu_{l_{y}}, l_{y}, l_{x}\right\}$ are the corresponding Gibbs distributions that guarantee the factorization property (2).

Proof. The proof is the similar to the proof of the Theorem 3.3 in [5].

Let $\mu \in F$ and $\left\{\mu_{l_{x}}, x \in V^{l_{0}}\right\}$ be the corresponding Gibbs distributions on $\Gamma_{l_{x}}^{k}, x \in V^{l_{0}}$. Consider the distribution $\mu_{l_{x}}\left(\sigma\left(l_{x}\right)\right)$ of the configuratio $\sigma\left(l_{x}\right)$ with respect to $\mu_{l_{x}}$ writing it in the form $\mu_{l_{x}}\left(\sigma\left(l_{x}\right)\right)=$ $Z^{-1} \exp \left(\sigma(x) \sigma(y) h_{x y, \sigma(x) \sigma(y)}\right)$, where $h_{x y, \sigma(x) \sigma(y)} \in R$ is the "effective" local external field at the edge $l_{x}$ induced by $\mu_{l_{x}}$ and $Z$ is the normalizing factor. Let $l_{x}=$ $\langle x, y\rangle, l_{y}=\langle y, z\rangle$, where $x \prec y \prec z$. It is evident that $\mu_{l_{x}}(\sigma(x), \sigma(y))=\sum_{\sigma(z) \in\{+1,-1\}} \mu_{l_{x}}\{\sigma(x), \sigma(y), \sigma(z)\}$, that is

$$
\begin{aligned}
& L^{-1} \exp \left(\sigma(x) \sigma(y) h_{x y, \sigma(x) \sigma(y)}\right) \\
& \quad=\sum_{\sigma(z) \in\{+1,-1\}} \exp \left(\sigma(y) \sigma(z) h_{y z, \sigma(y) \sigma(z)}\right. \\
& \quad+\beta J_{p} \sigma(x) \sigma(y) \sigma(y) \sigma(z) \\
& \left.\quad+\beta J_{t} \sigma(x) \sigma(y) \sigma(z)+\beta J_{1} \sigma(y)(\sigma(x)+\sigma(z))\right) .
\end{aligned}
$$

Then for fixed $\sigma(x)= \pm 1$ and $\sigma(y)= \pm 1$, we obtain the following recurrent equations:

$$
\text { (i) } \begin{aligned}
& L^{-1} \exp \left(h_{x y,++}\right) \\
= & \exp \left(h_{y z,++}+\beta J_{p}+\beta J_{t}+2 \beta J_{1}\right) \\
+ & \exp \left(-h_{y z,+-}-\beta J_{p}-\beta J_{t}\right),
\end{aligned}
$$

$$
\begin{aligned}
& \text { (ii) } \quad L^{-1} \exp \left(-h_{x y,+-}\right) \\
& =\exp \left(-h_{y z,-+}+\beta J_{p}-\beta J_{t}-2 \beta J_{1}\right) \\
& +\exp \left(h_{y z,--}+\beta J_{t}-\beta J_{p}\right), \\
& \text { (iii) } \quad L^{-1} \exp \left(-h_{x y,-+}\right)=\exp \left(h_{y z,++}-\beta J_{t}-\beta J_{p}\right) \\
& +\exp \left(-h_{y z,+-}+\beta J_{t}+\beta J_{p}-2 \beta J_{1}\right), \\
& \text { (iv) } \quad L^{-1} \exp \left(h_{x y,--}\right)=\exp \left(-h_{y z,-+}+\beta J_{t}-\beta J_{p}\right) \\
& +\exp \left(h_{y z,--}-\beta J_{t}+\beta J_{p}+2 \beta J_{1}\right) .
\end{aligned}
$$

In this paper, our main aim is to solve the system of equations in (4). Therefore, there are three cases when this system of equations is solvable:

1. $h_{++}=h_{-+}=h_{1}(y, z)$ and $h_{--}=h_{+-}=h_{2}(y, z)$;

2. $h_{++}=-h_{--}=h_{1}(y, z)$ and $h_{+-}=-h_{-+}=$ $h_{2}(y, z)$

3. $h_{++}=h_{+-}=h_{1}(y, z)$ and $h_{--}=h_{-+}=h_{2}(y, z)$.

For each of these cases we will define the transformation:

$$
\boldsymbol{F}=\left(F_{1}, F_{2}\right): R^{2} \rightarrow R^{2}
$$

with $h_{1}^{\prime}=F_{1}\left(h_{1}, h_{2}\right)$ and $h_{2}^{\prime}=F_{2}\left(h_{1}, h_{2}\right)$. The fixed points of Eq. (5) such that $\boldsymbol{h}=\boldsymbol{F}(\boldsymbol{h})$, where $\boldsymbol{h}=\left(h_{1}, h_{2}\right)$, describe translation-invariant phases of the model (1).

\section{Markov random fields with memory 2: phase transitions}

In this section we consider the Gibbs states (phases) that correspond in probability theory to what is called Markov chains with memory length 2 for the Ising model obtained by Hamiltonian (1).

Case 1. Assume that $h_{++}=h_{-+}=h_{1}(y, z)=h_{1}$ and $h_{--}=h_{+-}=h_{2}(y, z)=h_{2}$. Then for an edge $l_{x}=\langle x, y\rangle$ with direct successor $l_{y}^{1}=\langle y, z\rangle$ and $l_{y}^{2}=\langle y, t\rangle$ using equations given in Eq. (4). From Eq. (4), if we divide $\frac{(\mathrm{i})}{(\text { iii) }}$ and $\frac{(\mathrm{ii})}{(\mathrm{iv})}$ then we produce following recurrent equations:

$$
\begin{aligned}
& h_{1}(x, y)=\log a+\frac{1}{2} \log \left(\frac{(b c)^{2} \mathrm{e}^{h_{1}(y, z)+h_{2}(y, z)}+1}{a \mathrm{e}^{h_{1}(y, z)+h_{2}(y, z)}+(b c)^{2}}\right. \\
& \left.\times \frac{(b c)^{2} \mathrm{e}^{h_{1}(y, t)+h_{2}(y, t)}+1}{a \mathrm{e}^{h_{1}(y, t)+h_{2}(y, t)}+(b c)^{2}}\right), \\
& h_{2}(x, y)=\log a+\frac{1}{2} \log \left(\frac{c^{2}+a b^{2} \mathrm{e}^{h_{1}(y, z)+h_{2}(y, z)}}{b^{2}+a c^{2} \mathrm{e}^{h_{1}(y, z)+h_{2}(y, z)}}\right. \\
& \left.\times \frac{c^{2}+a b^{2} \mathrm{e}^{h_{1}(y, t)+h_{2}(y, t)}}{b^{2}+a c^{2} \mathrm{e}^{h_{1}(y, t)+h_{2}(y, t)}}\right),
\end{aligned}
$$

where $\exp \left(2 \beta J_{1}\right)=a, \exp \left(\beta J_{p}\right)=b, \exp \left(\beta J_{t}\right)=c$ and $\beta=1 / T$.

Theorem 3.4. For any collection of the quantities $\left\{h_{l}, l \in L^{0}\right\}$ satisfying these recurrent equations there exists the Gibbs distribution $\mu \in F\left(\Gamma_{l_{0}}^{k}\right)$; moreover, the distribution is unique. 
If we choose as $h_{++}=h_{-+}=h_{1}(y, z)=h_{1}$ and $h_{--}=h_{+-}=h_{2}(y, z)=h_{2}$, then we obtain the transformation $\boldsymbol{F}=\left(F_{1}, F_{2}\right): R^{2} \rightarrow R^{2}$ with $h_{1}^{\prime}=F_{1}\left(h_{1}, h_{2}\right)$ and $h_{2}^{\prime}=F_{2}\left(h_{1}, h_{2}\right)$, where

$$
\begin{aligned}
& h_{1}^{\prime}=\log \left(\frac{a\left((b c)^{2} \mathrm{e}^{h_{1}+h_{2}}+1\right)}{a \mathrm{e}^{h_{1}+h_{2}}+(b c)^{2}}\right), \\
& h_{2}^{\prime}=\log \left(\frac{a\left(c^{2}+a b^{2} \mathrm{e}^{h_{1}+h_{2}}\right)}{b^{2}+a c^{2} \mathrm{e}^{h_{1}+h_{2}}}\right) .
\end{aligned}
$$

The fixed points $\boldsymbol{h}=\boldsymbol{F}(\boldsymbol{h})$ of Eq. (5), where $\boldsymbol{h}=\left(h_{1}, h_{2}\right)$, describe translation-invariant phases of the model (1). Let us investigate the fixed points of the dynamic system (6):

$$
\begin{aligned}
& h_{1}=\log \left(\frac{a\left((b c)^{2} \mathrm{e}^{h_{1}+h_{2}}+1\right)}{a \mathrm{e}^{h_{1}+h_{2}}+(b c)^{2}}\right), \\
& h_{2}=\log \left(\frac{a\left(c^{2}+a b^{2} \mathrm{e}^{h_{1}+h_{2}}\right)}{b^{2}+a c^{2} \mathrm{e}^{h_{1}+h_{2}}}\right) .
\end{aligned}
$$

If we choose as $\exp \left(h_{1}\right)=z_{1}$ and $\exp \left(h_{2}\right)=z_{2}$, then we get the following equations:

$$
z_{1}=\frac{a\left((b c)^{2} z_{1} z_{2}+1\right)}{a z_{1} z_{2}+(b c)^{2}}, \quad z_{2}=\frac{a\left(a b^{2} z_{1} z_{2}+c^{2}\right)}{a c^{2} z_{1} z_{2}+b^{2}} .
$$

Assume that $u=z_{1} z_{2}$. If we multiply the identities in (7) then we obtain

$$
g(u):=\frac{a^{2}\left((b c)^{2} u+1\right)\left(a b^{2} u+c^{2}\right)}{\left(a u+(b c)^{2}\right)\left(a c^{2} u+b^{2}\right)}=u .
$$

We obtain

$$
\begin{aligned}
& a^{2} c^{2}+\left(a^{3} b^{2}-b^{4} c^{2}+a^{2} b^{2} c^{4}\right) u \\
& \quad+\left(-a b^{2}+a^{3} b^{4} c^{2}-a b^{2} c^{4}\right) u^{2}-a^{2} c^{2} u^{3}=0 .
\end{aligned}
$$

Let us note that if there is more than one positive solution for the equations in (7), then there is more than one translation-invariant Gibbs measure corresponding to these solutions. We say that a phase transition occurs for the model (1), if Eq. (9) has more than one positive solution.

Case 2. Now, let us consider the second special case: $h_{++}=h_{+-}=h_{1}(y, z) ; h_{--}=h_{-+}=h_{2}(y, z)$. Similar to Case 1, from Eq. (4), if we divide $\frac{(\mathrm{i})}{(\text { ii) }}$ and $\frac{\text { (iv) }}{\text { (iii) }}$ and if we choose as $\exp \left(h_{1}\right)=z_{1}$, $\exp \left(h_{2}\right)=z_{2}$, then we can get the following equations:

$$
\begin{aligned}
& z_{1}=\frac{a z_{2}\left[a(b c)^{2} z_{1}^{2}+1\right]}{z_{1}\left(b^{2}+a c^{2} z_{2}^{2}\right)}, \\
& z_{2}=\frac{a z_{1}\left(c^{2}+a b^{2} z_{2}^{2}\right)}{z_{2}\left[(b c)^{2}+a z_{1}^{2}\right]} .
\end{aligned}
$$

From the first equation in (10), we have $z_{1}^{2}=$ $\frac{a z_{2}}{\left(b^{2}+a c^{2} z_{2}^{2}-(a b c)^{2} z_{2}\right)}$ and substituting it to squared second one we get the following equation:

$$
\begin{aligned}
& -a^{3} b^{2} c^{4} v+a^{5} b^{2} c^{6} v^{2}+\left(-2 a^{4} b^{4} c^{2}-a^{5} c^{6}\right) v^{3} \\
& \quad+\left(2 a^{6} b^{4} c^{4}+b^{8} c^{4}\right) v^{4} \\
& \quad+\left(-a^{5} b^{6}+2 a^{2} b^{4} c^{2}-2 a^{6} b^{2} c^{4}-2 a^{2} b^{8} c^{6}\right) v^{5} \\
& \quad+\left(a^{4}+a^{7} b^{6} c^{2}-2 a^{4} b^{4} c^{4}+2 a^{2} b^{6} c^{6}+a^{4} b^{8} c^{8}\right) v^{6}
\end{aligned}
$$

$$
\begin{aligned}
& +\left(-a^{7} b^{4} c^{2}+2 a^{4} b^{2} c^{4}-2 a^{4} b^{6} c^{8}\right) v^{7}+a^{4} b^{4} c^{8} v^{8} \\
& =0
\end{aligned}
$$

where for brevity assume $z_{2}=v$. In this case, if positive solutions of the last equation are more than one, then there exists phase transition. Also, the solutions of the last equation describe the extreme Gibbs measures with memory 2 corresponding to the model (1).

Case 3. Lastly, let us consider the third special case $h_{++}=-h_{--}=h_{1}(y, z)=h_{1} ; h_{+-}=-h_{-+}=$ $h_{2}(y, z)=h_{2}$. Again similar to Case 1, from Eq. (4), if we divide $\frac{\text { (i) }}{\text { (iv) }}$ and $\frac{\text { (iii) }}{\text { (ii) }}$ and if we choose as $\exp \left(h_{1}\right)=z_{1}$ and $\exp \left(h_{2}\right)=z_{2}$, we have

$$
z_{1}=\frac{z_{1}\left[a(b c)^{2} z_{1} z_{2}+1\right]}{z_{2}\left(c^{2} z_{1} z_{2}+a b^{2}\right)}, \quad z_{2}=\frac{z_{1}\left[a z_{1} z_{2}+(b c)^{2}\right]}{z_{2}\left(b^{2} z_{1} z_{2}+a c^{2}\right)} .
$$

From (11), we have $z_{2}=\frac{a(b c)^{2} z_{1} z_{2}+1}{c^{2} z_{1} z_{2}+a b^{2}}$ and $z_{2}^{2}=$ $\frac{z_{1}\left[a z_{1} z_{2}+(b c)^{2}\right]}{b^{2} z_{1} z_{2}+a c^{2}}$. If we multiply the last equation by $z_{2}$ and assume that $z_{1} z_{2}=x$, then we obtain the following equation: $x=\left(\frac{a(b c)^{2} x+1}{c^{2} x+a b^{2}}\right)^{3}\left(\frac{b^{2} x+a c^{2}}{a x+(b c)^{2}}\right)=g(x)$.

We should obtain the fixed point of the function $g$. In order to describe the solution of the system of nonlinear equations in (11), we should analyze the function $g(u)$. Let us take the first derivative of $g(x)$;

$$
g^{\prime}(x)=\frac{c^{2}\left(1+a b^{2} c^{2} x\right)^{2}\left(A+B x+C x^{2}\right)}{\left(b^{2} c^{2}+a x\right)^{2}\left(a b^{2}+c^{2} x\right)^{4}},
$$

where $A=a b^{2}\left[b^{4}-3 c^{4}+a^{2}\left(-1+3 b^{4} c^{4}\right)\right], \quad B=$ $2\left[-b^{4}+a^{4} b^{4}+2 a^{2}\left(-1+b^{8}\right)\right] c^{2}$ and $C=a b^{2}\left[-3+b^{4} c^{4}+\right.$ $\left.a^{2}\left(3 b^{4}-c^{4}\right)\right]$.

Due to the positivity of $\frac{c^{2}\left(1+a b^{2} c^{2} x\right)^{2}}{\left(b^{2} c^{2}+a x\right)^{2}\left(a b^{2}+c^{2} x\right)^{4}}$, we study the sign of $A+B x+C x^{2}$. In order to study the fixed points of Eq. (5) such that $\boldsymbol{h}=\boldsymbol{F}(\boldsymbol{h})$, we have obtained $\boldsymbol{h}=\left(h_{1}, h_{2}\right)=\left(\log \left(z_{1}\right), \log \left(z_{2}\right)\right)$. Thus, for the third case we will describe the set of the extreme Gibbs measures with memory 2 corresponding to the model (1).

\section{Conclusions}

In previous works [4, 5], for given Hamiltonians we obtained the Gibbs states that correspond in probability theory to what are called Markov chains with memory length 2 . In this paper, we have studied the extreme Gibbs measures corresponding to Hamiltonian (1). We have considered three types of transformation $\boldsymbol{F}=$ $\left(F_{1} ; F_{2}\right): R^{2} \rightarrow R^{2}$. Since we will have some complicated formulae for the coefficients and the solutions, we do not present the solution here. The proofs and results will be considered in more detail later on in future publications. Also, the study of periodic the Gibbs measures will be considered in more detail later on in future publications.

\section{Acknowledgments}

This work is supported by TUBITAK, project number: $109 \mathrm{~T} 678$ 


\section{References}

[1] J.W. Gibbs, Elementary Principles in Statistical Mechanics, Yale University Press, New Haven 1902.

[2] L. Boltzmann, Lectures on the Theory of Gases, Gauthier-Villars, Paris, tome I 1902, tome II 1905, reedition Jean Gabay, Paris 1987 (in French).

[3] N. Ganikhodjaev, H. Akın, S. Uğuz, S. Temir, J. Concrete Appl. Math. 9, 26 (2011).

[4] N. Ganikhodjaev, H. Akın, S. Uğuz, S. Temir, Phase Transit. 84, 1045 (2011)

[5] N. Ganikhodjaev, H. Akın, S. Uğuz, S. Temir, J. Stat. Mech. 2011, P03025 (2011)
[6] J. Vannimenus, Z. Phys. B 43, 141 (1981)

[7] M. Fannes, A. Verbeure, Commun. Math. Phys. 96, 115 (1984)

[8] H.O. Georgii, Gibbs Measures and Phase Transitions, de Gruyter Stud. Math., Vol. 9, Walter de Gruyter, Berlin 1988.

[9] Ch.J. Preston, Gibbs States on Countable Sets, Cambridge Univ. Press, Cambridge 1974.

[10] F. Spitzer, Ann. Probab. 3, 387 (1975) 